\title{
Métodos para avaliação da qualidade de comprimidos de cinarizina como proposta de monografia farmacopeica
}

\section{Methods for assessing the quality of cinarizin tablets as a proposed pharmacopeic monograph}

\section{Métodos para la evaluación de la calidad de comprimidos de cinarizina como propuesta de monografía farmacopeica}

\author{
BERTOL, Charise Dallazem ${ }^{1,2}$. FREITAS, Alessandra Rauber de ${ }^{1}$. ZANANDREA, Tatieli ${ }^{1}$. ARESI, Nicolas ${ }^{1}$. \\ ANZOLIN, Ana Paula2. GRANDO, Luciana Grazziotin Rossato ${ }^{1,3}$. \\ ${ }^{1}$ ICB - Instituto de ciências biológicas, Faculdade de Farmácia, Universidade de Passo Fundo, Passo \\ Fundo, Brasil. 2PPGEH - Programa de Pós-Graduação em Envelhecimento Humano, Universidade de \\ Passo Fundo, Passo Fundo, Brasil. 3PPGBioexp - Programa de Pós-graduação em \\ Bioexperimentação, Universidade de Passo Fundo, Passo Fundo, Brasil. \\ *charise@upf.br
}

Resumo. A cinarizina é amplamente prescrita para desordens vestibulares. Não há monografia para avaliar a qualidade dos comprimidos nas farmacopeias. Este estudo tem como objetivo desenvolver métodos para avaliar a qualidade de comprimidos referência $(R)$, genérico $(G)$, e similar (S) de cinarizina. Para o doseamento foi validado um método por espectrofotometria no UV, comparado com o método volumétrico. Os comprimidos R, $\mathrm{G}$ e $\mathrm{S}$ foram submetidos aos ensaios gerais de controle de qualidade. A solubilidade em pH 1,2, 4,5 e 6,8 foi determinada. Na validação comparou-se a performance dos aparatos pá (velocidades 50 e $75 \mathrm{rpm}$ ) e cesta (50 e $100 \mathrm{rpm}$ ) na dissolução de R, G e S. O doseamento foi validado e mostrou-se preciso, exato, linear, robusto e específico. Os comprimidos R, G e S apresentaram conformidade no doseamento, peso médio, dureza, friabilidade, desintegração e, uniformidade. O método de dissolução demonstrou linearidade, precisão, exatidão e especificidade em pH 1,2, em $254 \mathrm{~nm}$. A dissolução de R, G e S foi maior que $70 \%$ em 30 minutos. O aparato pá a 75 rpm é a condição que discrimina melhor as formulações, pois nessa condição $\mathrm{G}$ e $S$ não são equivalentes farmacêuticos ao R. Os métodos desenvolvidos apresentam a confiabilidade requerida para análise farmacopeica.

Palavras-chave: Validação. Espectrofotometria. Dissolução. Fator de Similaridade. Equivalência Farmacêutica.

Abstract. Cinnarizine is widely prescribed for vestibular disorders. There is no monograph to evaluate the quality of the tablets in pharmacopoeias. To develop methods to evaluate the quality of reference (R), generic $(\mathrm{G})$, and similar (S) cinarizine tablets. For the assay a UV spectrophotometric method was validated, compared to the volumetric method. $\mathrm{R}, \mathrm{G}$ and $\mathrm{S}$ were submitted to the general quality tests. The solubility ( $\mathrm{pH} 1.2,4.5$ and 6.8) was determined. In the validation, the performance of the paddle (velocity 50 and $75 \mathrm{rpm}$ ) and basket (50 and $100 \mathrm{rpm}$ ) apparatus in the dissolution of R, G and $\mathrm{S}$ were compared. The assay was validated and demonstrates accuracy, precision, linearity, robustness and specificity. R, G and S presented conformity in the assay, average weight, hardness, friability, disintegration and uniformity. The dissolution method demonstrated linearity, precision, accuracy and specificity at $\mathrm{pH} 1.2$, at $254 \mathrm{~nm}$. The dissolution of $\mathrm{R}, \mathrm{G}$ and $\mathrm{S}$ was greater than $70 \%$ in 30 minutes. The apparatus paddle at $75 \mathrm{rpm}$ is the condition that better discriminates the formulations, since in this condition $G$ and $S$ are not pharmaceutical equivalents to $R$. The developed method present the reliability required for a pharmacopoeial analysis.

Keywords: validation, spectrophotometry, dissolution, similarity factor, pharmaceutical equivalence.

Resumen. La cinarizina es prescrita para desórdenes vestibulares. Actualmente no se cuenta con una monografía para evaluar la calidad de los comprimidos en las farmacopeas. Desarrollar métodos para evaluar la calidad de los comprimidos de referencia $(R)$, genérico $(G)$, y similar $(S)$ de cinarizina. Para la determinación de cinarizina fue validado un método por espectrofotometría UV comparado con el método volumétrico. $R, G$ y $\mathrm{S}$ fueron sometidos a ensayos de control de calidad. La solubilidad se determinó en pH 1,2, 4,5, 6,8. En la validación se comparó el desempeño de los aparatos paleta (velocidades 50 y 75 rpm) y cesta (50 y $100 \mathrm{rpm}$ ) en la disolución de los comprimidos. La determinación de cinarizina fue validada y se mostró precisa, exacta, lineal, robusta y específica. R, G y S presentaron conformidad en la determinación, peso medio, dureza, friabilidad, desintegración y uniformidad. El método de disolución demostró linealidad, precisión, exactitud y especificidad en $\mathrm{pH} 1,2$, en $254 \mathrm{~nm}$. La disolución de R, G y S fue mayor que $70 \%$ en 30 minutos. El aparato paleta a $75 \mathrm{rpm}$ es la condición que discrimina mejor las formulaciones, pues en esa condición G y $\mathrm{S}$ no son equivalentes a R. Los métodos desarrollados presentan la confiabilidad para un análisis farmacopeico. Palabras-clave: Validación. Espectrofotometría. Disolución. Factor de similitud. Equivalencia farmacéutica. 


\section{Introdução}

A cinarizina foi sintetizada em $1955^{(1)}$ e, em 1957 foi comercializada como comprimidos de 25 e $75 \mathrm{mg}$. É uma base fraca (pka 1,95 e 7,5), quimicamente conhecida como (E)-1-(difenilmetil)-4-(3-fenil-2-propenil) piperazina (Figura 1), possui maior solubilidade em $\mathrm{pH}$ ácido devido a protonação dos dois nitrogênios em $\mathrm{pH}$ ácido ${ }^{(2,3)}$.

Figura 1. Estrutura química da cinarizina.

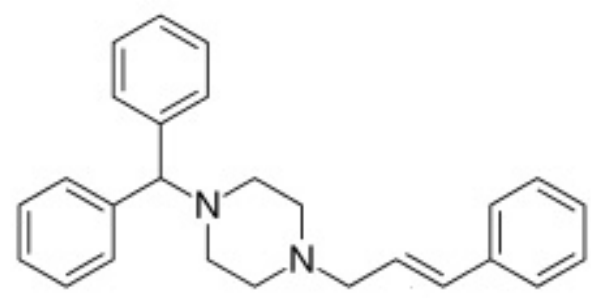

A cinarizina é um antagonista de cálcio, bloqueando seletivamente a entrada deste íon na célula, através das membranas celulares, inibindo o processo contrátil da musculatura vascular lisa, ocasionando uma vasodilatação arteriolar e redução da resistência periférica. É um fármaco vasodilatador periférico e cerebral, anti-histamínico $\mathrm{H} 1$, com ação anti-serotoninérgica, anti-dopaminérgica, antiemético e antivertiginoso, empregado no controle de distúrbios vasculares e, para prevenir e tratar distúrbios de movimento $(4,5)$.

A cinarizina é amplamente prescrita no Brasil, comercializada em farmácias e, está presente na relação municipal de medicamentos (REMUME) de diversos municípios, porém não é aprovada pelo FDA (Food and Drug Administration), devido a uma associação com a prevalência de Parkinson ${ }^{(6-9)}$.

A monografia para avaliação da matéria-prima de cinarizina está presente nas Farmacopeias Britânica, Europeia e Portuguesa e ausente nas Farmacopeias Americana e Brasileira. O projeto para a monografia de comprimidos de cinarizina está disponível no site da Farmacopeia Britânica para possível publicação em 2019(10), entretanto, para a análise de comprimidos, é utilizada cromatografia líquida de alta eficiência.

Dessa forma, esse trabalho objetivou desenvolver métodos simples, para avaliar a qualidade de comprimidos de cinarizina disponíveis no mercado brasileiro, utilizando a espectrofotometria na região do ultravioleta (UV). Para isso, foram utilizados os testes gerais disponíveis na farmacopeia para qualquer formulação e, foram desenvolvidas e validadas metodologias para doseamento e para o ensaio de dissolução.

\section{Materiais e Métodos}

\section{Amostras}

A matéria-prima de cinarizina (Lote 0709053604, La Nova Chen) foi utilizada como substância química de referência (SQR).

Para os ensaios de avaliação dos comprimidos foram utilizados: medicamento referência (R) (Stugeron ${ }^{\circledR} 75 \mathrm{mg}$ ), genérico (G), e similar (S) contendo $75 \mathrm{mg}$ de cinarizina.

Os excipientes descritos na bula das formulações foram: R: amido, lactose, estearato de magnésio, polivinilpirrolidona ( $p v p)$, sacarose, talco e corante; G: amido, lactose, estearato de magnésio, pvp, sacarose e talco; S: celulose microcristalina, aerosil, estearato de magnésio, lactose, carboxi-metilcelulose sódica, lauril sulfato de sódio, crospovidona, amido, pvp, talco, corante e álcool etílico.

\section{Método Titulométrico}

O teor da SQR foi determinado por titulação de acordo com a Farmacopeia Britânica. Para isso, foram pesados $0,150 \mathrm{~g}$ de cinarizina dissolvida em $50 \mathrm{~mL}$ de uma mistura contendo ácido acético anidro e etil metil cetona (1:7). Esta solução foi titulada em titulador Titrino 702 SM (METROHM/ Pensalab) com ácido perclórico $0,1 \mathrm{~mol} / \mathrm{L}$ SV (padronizado frente ao biftalato de potássio) determinando o ponto final com potenciômetro. A titulação em branco foi realizada para correções. O cálculo de teor da SQR foi efetuado considerando que cada $\mathrm{mL}$ de ácido perclórico 0,1 M equivale a 18,43 mg de cinarizina $^{(2)}$.

O método titulométrico também foi aplicado para os comprimidos $\mathrm{R}$ nas mesmas condições acima, para comparar com o método espectrofotométrico desenvolvido. Os resultados de teor obtidos para os comprimidos $\mathrm{R}$ foram comparados pelo teste $t$ de student $(p<0,05)$.

\section{Desenvolvimento e Validação de Método de Doseamento por Espectrofotometria na Região do Ultravioleta / Visível (UV/VIS)}

O metanol foi utilizado para o preparo das amostras, pois a cinarizina é facilmente solúvel neste solvente ${ }^{(2)}$. A SQR foi preparada na concentração de $32,0 \mu \mathrm{g} / \mathrm{mL}$, e, foi realizada uma varredura na região do UV/Vis em espectrofotômetro Lambda 20 (Perkin Elmer) na faixa de comprimento de onda de 200-400 nm observando os picos máximos de absorção.

A validação do método contemplou os parâmetros: precisão e exatidão, especificidade, linearidade e intervalo, robustez e, foi realizada utilizando a $\mathrm{SQR}^{(11)}$. O método desenvolvido foi aplicado para análise dos comprimidos.

Precisão: Foi investigada através da repetibilidade (precisão intra-dia), onde seis amostras de SQR de igual concentração teórica $(40,0 \mu \mathrm{g} / \mathrm{mL})$ foram preparadas durante o mesmo dia e sob as mesmas condições experimentais. Os resultados foram expressos como desvio padrão relativo (DPR).

Exatidão: Foi verificada através de três concentrações diferentes de $\mathrm{SQR}$, contemplando o intervalo linear do método que foi de $12,0,20,0$ e $36,0 \mu \mathrm{g} / \mathrm{mL}$, em triplicata para cada concentração $(n=9)$. A exatidão foi expressa pela relação entre a concentração média determinada experimentalmente e a concentração teórica correspondente, conforme a equação 1 :

Equação 1

Recuperação $(\%)=($ concentração experimental/concentração teórica $) \times 100$

Linearidade: Foi determinada através da construção de três curvas de calibração, nas concentrações de 8,0, 16,0, 24,0, 32,0 , e $40,0 \mu \mathrm{g} / \mathrm{mL}$ de SQR. As absorbâncias foram plotadas versus as concentrações utilizadas para obter a curva de calibração. Os resultados obtidos foram submetidos à análise de regressão pelo método dos mínimos quadrados para obtenção da equação da reta e do coeficiente de correlação e, análise estatística por ANOVA ( $\alpha=5 \%$ ).

Especificidade: Foi avaliada pela análise de uma solução placebo composta por lactose, amido, sacarose, talco e estearato de magnésio que correspondem aos excipientes presentes nos comprimidos R. A habilidade para medir especificadamente o analito foi examinada pela ausência de picos de absorção da solução placebo no comprimento de onda utilizado para estimar a resposta da cinarizina, em uma solução contendo $2,5 \mathrm{mg} / \mathrm{mL}$ de excipientes em metanol.

Robustez: Foi avaliada através da estabilidade de soluções de SQR de cinarizina $(8,0-40,0 \mu \mathrm{g} / \mathrm{mL})$ no diluente (metanol) mantida em temperatura ambiente por $48 \mathrm{~h}$, comparando com uma curva recém-preparada.

\section{cinarizina}

Controle de qualidade dos comprimidos de

Os métodos gerais foram utilizados de acordo com a Farmacopeia Brasileira(12).

Doseamento: O método titulométrico(2) foi utilizado apenas para o doseamento dos comprimidos $\mathrm{R}$ para fins de comparação de métodos. Para as outras amostras ( $G$ e $S$ ) foi utilizado o método desenvolvido e validado. Para o doseamento, 
20 comprimidos $\mathrm{R}$, G ou S foram triturados e, uma alíquota equivalente a $0,150 \mathrm{~g}$ de cinarizina foi retirada, diluída em metanol até ser obtida uma solução na concentração de 24,0 $\mu \mathrm{g} / \mathrm{mL}$ em triplicata. A absorbância desta solução foi lida em $250 \mathrm{~nm}$. A determinação da concentração foi obtida a partir de uma curva de calibração da SQR preparada no mesmo dia.

Peso médio: Foi obtido a partir da pesagem de 20 comprimidos R, G e S, em balança analítica (Ohaus, Marte), calcularam-se a média, limites superior e inferior ${ }^{(12)}$.

Dureza: Foi utilizado durômetro manual (Nova Ética) em 10 comprimidos $\mathrm{R}$, $\mathrm{G}$ e $\mathrm{S}^{(12)}$.

Friabilidade: Foi determinada em friabilômetro, modelo NT240 (Nova Ética) em 10 comprimidos R, G e S. Foi determinada a massa inicial e a final, e calculado o percentual de perda de massa ${ }^{(12)}$.

Desintegração: Foram utilizados seis comprimidos de cada amostra em desintegrador (N480, Nova Ética), utilizando água a $37^{\circ} \mathrm{C}$. O movimento das cestas foi cessado após todos os comprimidos estarem completamente desintegrados(12)

Uniformidade de doses unitárias: Foi determinada pelo método de variação de peso ${ }^{(12)}$. Foram pesados exatamente e individualmente, 10 comprimidos $R$, $\mathrm{G}$ e $\mathrm{S}$ e, foi estimado o conteúdo do componente ativo em cada unidade, utilizando a equação 2 :

\section{Equação 2}

$x i=p i \times A / P$

Onde pi são os pesos individuais das unidades, $A$ é a quantidade de componente ativo, expressa em porcentagem da quantidade declarada, determinada no doseamento, e $P$ é o peso médio das unidades utilizadas no doseamento.

Após calculou-se o valor de aceitação (VA), levando em consideração as equações do caso 1 , quando a média de teor estimada na monografia é $\leq 101,5 \%$.

\section{dissolução \\ Desenvolvimento e validação de ensaio de \\ No desenvolvimento do teste de dissolução(11,13), a} escolha dos meios de dissolução, quantidade, aparato e velocidade de rotação, foram baseadas na solubilidade da cinarizina.

Solubilidade: Foram realizadas varreduras de 200 a $400 \mathrm{~nm}$ de soluções de SQR (não saturadas) em diferentes $\mathrm{pH}$, para verificar os comprimentos de onda de absorção máxima. A solubilidade da cinarizina foi determinada através da adição de quantidades crescentes de cinarizina (SQR) em soluções de pH 1,2, 4,5 e 6,8, até a obtenção de soluções saturadas, que foram filtradas e medidas espectrofotometricamente. A absorbância das soluções não saturadas também foi medida.

Linearidade: Foi determinada através da construção de três curvas de calibração, compostas por cinco concentrações equivalentes a 5,0,10,0,15,0,20,0 e 25,0 $\mu \mathrm{g} / \mathrm{mL}$ de SQR. As absorbâncias foram plotadas versus as concentrações utilizadas para obter a curva de calibração. Os resultados foram submetidos à análise de regressão pelo método dos mínimos quadrados para obtenção da equação da reta e do coeficiente de correlação e análise estatística por ANOVA $(\alpha=5 \%)$.

Precisão: Avaliada através da repetibilidade (precisão intra-dia, análises no mesmo dia) e precisão interdia (em dias diferentes), onde seis amostras de igual concentração teórica $(25,0 \mu \mathrm{g} / \mathrm{mL})$ foram analisadas sob as mesmas condições experimentais. Os resultados foram expressos como DPR.

Exatidão: Foi verificada através de três concentrações de SQR, contemplando o intervalo linear do método que foram de 17,5 $\mu \mathrm{g} / \mathrm{mL}$ (baixa), 22,5 $\mathrm{gg} / \mathrm{mL}$ (média) e $27,5 \mu \mathrm{g} / \mathrm{mL}$ (alta), em triplicata para cada concentração. A recuperação (\%) foi calculada utilizando a equação 1 .
Especificidade: Foram preparadas soluções placebo contendo os excipientes em separado das formulações $R, G$ e $S$ na cuba de dissolução contendo $\mathrm{HCl} 0,1 \mathrm{~mol} / \mathrm{L}$ e desta retirouse uma alíquota após $60 \mathrm{~min}$ e foi feita a varredura no espectrofotômetro UV na faixa de $200-400 \mathrm{~nm}$.

\section{Obtenção dos perfis de dissolução de $R, \mathbf{G}$ e S:}

Os ensaios foram realizados para todas as amostras, em dissolutor Nova Ética, modelo 299/6. Foi utilizado $\mathrm{HCl} \mathrm{0,1} \mathrm{mol/L}$ pH 1,2 como meio de dissolução e dois aparatos, cestas e pás, nas velocidades de 100 e $50 \mathrm{rpm}$ e 75 e $50 \mathrm{rpm}$, respectivamente. Para cada cuba, foram utilizados $900 \mathrm{~mL}$ do meio, na temperatura de $37^{\circ} \mathrm{C}\left( \pm 0,5^{\circ} \mathrm{C}\right) . \mathrm{O} \mathrm{pH}$ foi medido em potenciômetro modelo DM 20 (Digimed) e, o meio de dissolução foi desaerado em banho de ultrassom modelo USC $2850 \mathrm{~A}$ (Ultrasonic Cleaner Thornton - Unique). Amostras de $10 \mathrm{~mL}$ foram coletadas nos tempos de 5, 10, 15, 20, 25, 30, 40, 50 e 60 minutos, sendo que em cada tempo, o meio de dissolução foi reposto. As alíquotas retiradas foram diluídas até que as absorbâncias ficassem dentro do intervalo linear do método de 5,0 a $25,0 \mu \mathrm{g} / \mathrm{mL}$. As absorbâncias das soluções foram medidas em espectrofotômetro Ultravioleta/Visível (UV/Vis) modelo Lambda 20 (Perkin Elmer). Foi calculado o teor de cinarizina dissolvida no meio, comparando as leituras obtidas com uma curva de calibração de SQR preparada no dia das análises. Os perfis de dissolução foram obtidos a partir da plotagem do fármaco dissolvido (\%) versus o tempo (min).

Para avaliar a semelhança entre os perfis de dissolução, foi utilizado o modelo matemático independente fator de similaridade (f2 - equação 3$)^{(13-15)}$.

O fator de similaridade (f2) é um logaritmo recíproco da transformação da raiz quadrada da soma do erro quadrado e indica a média da similaridade da porcentagem entre os dois perfis.

Equação 3

$$
f 2=50 \log \left\{\left(1+\frac{1}{n} \sum_{t=1}^{n}\left(R_{t}-T_{t}\right)^{2}\right)^{-0,5} \times 100\right\}
$$

Onde $\mathrm{n}$ é o número de tempos de amostras da dissolução, Rt e Tt são os percentuais individuais liberados em cada tempo t, para os dois perfis de dissolução.

A semelhança ou equivalência de dois perfis é observada quando os valores de f2 ficam entre 50 e $100^{(13-15)}$.

\section{Resultados e Discussão}

\section{Método Titulométrico}

A determinação do teor da SQR por titulação foi feita em triplicata, e foi obtida uma média de 99,97\% ( \pm D.P.R. de $2,11 \%$ ), estando de acordo com a especificação para a matériaprima de cinarizina de 99,0 a $101,0 \%{ }^{(2)}$

Desenvolvimento e Validação do Método de Doseamento por Espectrofotometria na Região do UV

Para a determinação do comprimento de onda utilizado no doseamento, após varredura de 200-400 nm no espectrofotômetro, foi selecionado o comprimento de onda de $250 \mathrm{~nm}$ o qual está relacionado a um dos máximos de absorção da cinarizina, sem que haja a interferência de excipientes e solventes (Figura 2).

A validação foi realizada utilizando a $S Q R$, e, posteriormente o método foi aplicado para os comprimidos. O método apresentou-se preciso com um DPR de 0,915\% de acordo com a legislação ${ }^{(11,16)}$.

Os percentuais de recuperação para as concentrações de $12,0,20,0$ e $36,0 \mu \mathrm{g} / \mathrm{mL}$ foram de $98,17 \%, 100,82 \%$ e $98,01 \%$, respectivamente, próximos a $100 \%$ em todos os casos, comprovando a exatidão do método.

A curva de calibração média obteve uma equação da reta de $y=0,0586 x-0,084$, sendo que o coeficiente de correlação 
médio foi de 0,9997, comprovando a linearidade do método, pois o critério mínimo aceitável do coeficiente de correlação é maior que $0,99^{(11)}$. A linearidade do método foi comprovada estatisticamente. Observou-se diferença significativa entre as concentrações (Fcal > Ftab; 494,53 > 3,48 ), regressão linear significativa (Fcal > Ftab, 1976,39> 4,96 ) e desvio de linearidade não significativo (Fcal < Ftab; $0,593<3,71)$, demonstrando a linearidade no intervalo testado $(8,0-40,0 \mu \mathrm{g} / \mathrm{mL})$.

Figura 2. A) Varredura de solução metanólica de SQR de cinarizina $(32,0 \mu \mathrm{g} / \mathrm{mL})$ em espectrofotômetro UV/VIS na faixa de 200 a $400 \mathrm{~nm}$. B) Varredura de solução placebo em espectrofotômetro UV/VIS na faixa de 200 a $400 \mathrm{~nm}$.

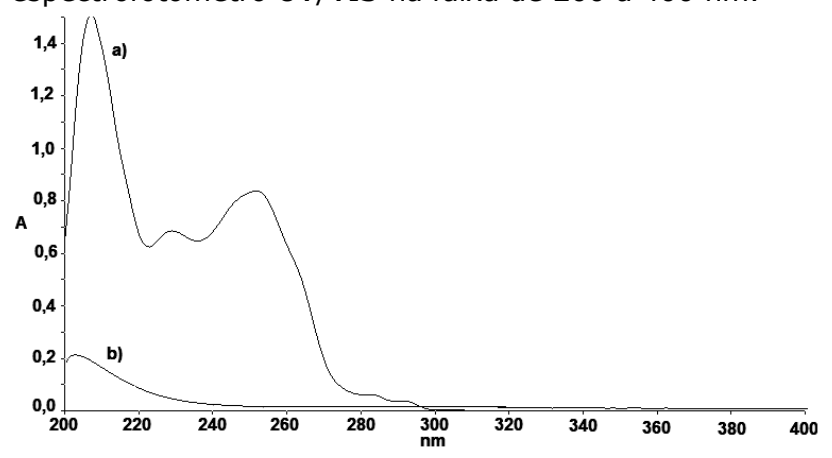

Não foram observados picos interferentes relacionados aos excipientes no comprimento de onda utilizado para as análises comprovando a especificidade do método (Figura 2).

A solução SQR de cinarizina foi estável por $48 \mathrm{~h}$, pois o teor manteve-se inalterado, assim como a resposta do método, mostrando que o método é robusto, ou seja, capaz de manter os mesmos resultados frente a pequenas variações.

\section{cinarizina}

Controle de qualidade dos comprimidos de

A formulação $R$ foi submetida aos ensaios de titulação e espectrofotometria na região do UV, pois para o doseamento da matéria-prima de cinarizina a Farmacopeia Britânica descreve a titulação ${ }^{(2)}$. Os valores médios de teor obtidos para os comprimidos $\mathrm{R}$, por meio dos ensaios de titulação e de espectrofotometria foram de $97,80 \%$ ( \pm D.P.R. 0,766\%) e 96,72 \% ( \pm D.P.R. 0,234\%), respectivamente. Não houve diferença estatística entre os resultados de teor obtidos por ambos os métodos ( $t$ calculado $<\mathrm{t}$ crítico com $\mathrm{p}<0,05)$.

Na tabela 1, encontram-se os resultados dos ensaios de qualidade obtidos para os comprimidos R, G e S.

Tabela 1. Ensaios de controle de qualidade para os comprimidos R, G e S.

\begin{tabular}{|c|c|c|c|c|c|c|}
\hline Amostra & Doseamento $^{1}$ & $\begin{array}{l}\text { Peso médio } \\
2\end{array}$ & Dureza $^{3}$ & $\begin{array}{l}\text { Friabilida } \\
\text { de }^{4}\end{array}$ & $\begin{array}{l}\text { Desintegração } \\
5\end{array}$ & Uniformidade $^{6}$ \\
\hline \multirow[t]{2}{*}{ R } & $96,72 \pm 0,234$ & 0,3044 & $5,33 \pm$ & 0,44 & $9^{\prime} 23^{\prime \prime}$ & $97,70 \pm 1,25$ \\
\hline & & & 4,42 & & & 9,22 \\
\hline \multirow[t]{2}{*}{ G } & $96,43 \pm 1,48$ & 0,3053 & $5,95 \pm$ & 0,62 & $12^{\prime} 44^{\prime \prime}$ & $96,41 \pm 0,55$ \\
\hline & & & 4,77 & & & 3,42 \\
\hline \multirow[t]{2}{*}{$\mathbf{S}$} & $91,09 \pm 1,03$ & 0,3623 & $5,55 \pm$ & 0,68 & $1^{\prime} 02^{\prime \prime}$ & $90,85 \pm 0,65 ;$ \\
\hline & & & 2,85 & & & 9,20 \\
\hline
\end{tabular}

1 Obtido pelo método validado por espectrofotometria na região do UV (média \% \pm DPR) $(\mathrm{n}=3) ;{ }^{2}$ em g $(\mathrm{n}=20) ;{ }^{3}$ (média kgf \pm DPR) $(\mathrm{n}=10) ;{ }^{4}$ perda de massa em $\% ;{ }^{5} \mathrm{em}$ minutos; 6 teor médio $\% \pm$ Desvio Padrão; Valor de Aceitação. DPR = Desvio Padrão Relativo.

No doseamento, considerando os limites estabelecidos de aceitação para outros comprimidos de liberação imediata, que normalmente variam de 90-110\% em diversas Farmacopeias, as amostras foram aprovadas.
Em relação ao peso médio, a variação individual permitida para comprimidos com mais de $250 \mathrm{mg}$ é de $\pm 5,0 \%$, e, neste ensaio todas as unidades ficaram dentro dos limites. No teste de dureza, as amostras foram aprovadas, já que a Farmacopeia não determina máximos e mínimos de dureza permitidos. $\mathrm{Na}$ friabilidade as amostras também foram aprovadas, pois a perda de massa foi menor que $1,5 \%$, assim como na desintegração, pois desintegraram em menos de 30 minutos. A amostra S apresentou a desintegração mais rápida, e isso pode estar relacionado ao excipiente crospovidona que é um desintegrante. $\mathrm{Na}$ uniformidade de doses unitárias pelo método de variação de peso, as amostras foram aprovadas, pois o VA foi menor que 15 em todos os $\operatorname{casos}^{(12)}$.

Validação do método de dissolução e ensaios de dissolução dos comprimidos de cinarizina

As soluções de cinarizina em $\mathrm{pH} 1,2$ obtiveram os máximos de absorção nos comprimentos de onda de 200 e 250 $\mathrm{nm}$, e, para os $\mathrm{pH} 4,5$ e 6,8 foi de $210 \mathrm{~nm}$.

A cinarizina apresenta baixa solubilidade em solventes aquosos e sua solubilidade é altamente dependente do $\mathrm{pH}$, sendo maior em pH 1,2, pois sendo uma base fraca, em meio ácido estará mais ionizada, e portanto mais solúvel (tabela 2). Dessa forma, optou-se por utilizar apenas o meio de $\mathrm{pH} \mathrm{1,2}$ para os ensaios de dissolução, pois a solubilidade deve ser assegurada considerando as condições sink, definida como o volume de meio três vezes maior que o volume necessário para saturar a solução(15).

Tabela 2. Resultados de solubilidade das soluções de cinarizina em diferentes $\mathrm{pH}$.

\begin{tabular}{lll}
\hline $\mathbf{p H}$ & Solubilidade & Classificação conforme Farmacopeia ${ }^{(12)}$ \\
\hline $\mathbf{1 , 2}$ & $85,59 \mu \mathrm{g} / \mathrm{mL}$ & Muito pouco solúvel \\
$\mathbf{4 , 5}$ & $0,68 \mu \mathrm{g} / \mathrm{mL}$ & Praticamente insolúvel ou insolúvel \\
$\mathbf{6 , 8}$ & $0,53 \mu \mathrm{g} / \mathrm{mL}$ & Praticamente insolúvel ou insolúvel \\
\hline
\end{tabular}

Outros trabalhos, com diferentes formulações de cinarizina, descrevem outras condições para a dissolução. Para

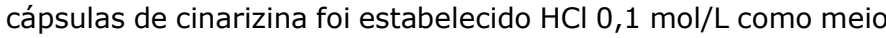
de dissolução, para assegurar a maior solubilidade do fármaco, aparato cesta a $100 \mathrm{rpm}$. As alíquotas retiradas dos meios de dissolução foram lidas em espectrofotômetro a $251 \mathrm{~nm}$, e duas das três cápsulas manipuladas, bem como o comprimido de referência, obtiveram uma liberação acima de 85\% em 30 $\min ^{(17)}$. Comprimidos de desintegração rápida de cinarizina foram produzidos com diferentes concentrações de Ac-di-sol (4 - $16 \%$ ) e de Cellactose $₫ 80(10-80 \%)$, e, avaliados utilizando - aparato pá a $50 \mathrm{rpm}, \mathrm{HCl} 0,1 \mathrm{~mol} / \mathrm{L}$ e leitura em espectrofotômetro ajustado em $254 \mathrm{~nm}$. Após 15 min todas as formulações apresentaram liberação acima de $80 \%{ }^{(18)}$. Comprimidos de dissolução rápida de cinarizina foram desenvolvidos variando concentrações de Cellactose $₫ 80$ (20$50 \%)$, celulose microcristalina (20-50\%), Tabletosse $®(20-$ $50 \%)$ e lactose spray dyer (20-50\%) e avaliados utilizando cesta a $50 \mathrm{rpm}$, fluído gástrico $\mathrm{pH} 1,2$ e leitura em espectrofotômetro em $254 \mathrm{~nm}$. Todas as formulações apresentaram um percentual de liberação acima de $80 \%$ em 30 min, e a Cellactose $\AA 80$ foi o excipiente com maior potencial para o desenvolvimento de comprimidos de liberação rápida(19). Comprimidos gastroresistentes flutuantes de cinarizina foram produzidos utilizando hidroxipropilmetilcelulose (de 4 diferentes graus de viscosidade), alginato de sódio ou polióxido de etileno e agente formador de gás (bicarnonato de sódio ou carbonato de cálcio) e, foram submetidos ao ensaio de dissolução utilizando cesta a 150 rpm, fluído gástrico $\mathrm{pH} \mathrm{1,2} \mathrm{e} \mathrm{análise} \mathrm{por} \mathrm{cromatografia}$ líquida de alta eficiência em $253 \mathrm{~nm}$. O lote produzido com hidroxipropilmetilcelulose K100LV mostrou uma maior biodisponibilidade em relação a uma suspensão de cinarizina devido a maior retenção no ambiente gastrintestinal, com uma liberação sustentada de até $24 \mathrm{~h}^{(20)}$. Dispersões sólidas de cinarizina contendo dois tipos de lipídeos Compritol 888 ATO $^{\circledR}$ e Gelucire $44 / 14^{\circledR}$ foram analisadas utilizando cesta a $100 \mathrm{rpm}$, 
fluído gástrico $\mathrm{pH} \mathrm{1,2} \mathrm{e} \mathrm{leitura} \mathrm{em} \mathrm{espectrofotômetro} \mathrm{em}$ $252 \mathrm{~nm}$. Os resultados demonstram que o perfil de liberação obtido depende do tipo e da quantidade de lipídeo utilizada, sendo que uma liberação mais prolongada foi obtida com cinarizina: Compritol (1:1) e cinarizina: Compritol $(1: 0,5)^{(21)}$.

A validação do método de dissolução prosseguiu utilizando $\mathrm{HCl} 0,1 \mathrm{~mol} / \mathrm{L} \mathrm{pH} \mathrm{1,2} \mathrm{e,} \mathrm{foi} \mathrm{avaliada} \mathrm{a} \mathrm{linearidade,}$ a precisão e a exatidão neste meio. Para garantir a seletividade da análise, sem a interferência de solventes e excipientes, foi selecionado $250 \mathrm{~nm}$ como comprimento de onda para as leituras. Após a construção de 3 curvas de calibração de soluções SQR no intervalo de 5,0 a $25,0 \mu \mathrm{g} / \mathrm{mL}$ foi obtida a seguinte equação da reta média ( $y=0,2225 \times$ $+0,488$ ) e um coeficiente de correlação de 0,9996, de acordo com a legislação(11). A linearidade foi comprovada estatisticamente, obtendo regressão linear significativa e desvio de linearidade não significativo, demonstrando a linearidade no intervalo testado. Para a precisão as 6 soluções na concentração de $25 \mu \mathrm{g} / \mathrm{mL}$ foram preparadas e lidas (250 nm), e o DPR foi de 1,40\% (para a precisão intradia) e de 1,07\% (para a precisão inter-dia), conforme especificação(11). Para a exatidão os percentuais de recuperação obtidos para as concentrações testadas $(17,5$, 22,5 e $27,5 \mu \mathrm{g} / \mathrm{mL}$ ) foram de: $99,97 \%$ (DPR de $\pm 0,814 \%$ ), $98,91 \%$ (DPR de $\pm 0,55 \%$ ) e $96,37 \%$ (DPR de $\pm 0,49 \%$ ), demonstrando a exatidão do método. Na especificidade, nenhuma das soluções placebos (obtidas com os excipientes do $R, G$ e S) demonstrou interferência no comprimento de onda utilizado.

Os perfis de dissolução dos comprimidos de cinarizina, utilizando os aparatos cesta e pás em diferentes velocidades de agitação, podem ser observados na figura 3 .

Figura 3. Perfis de dissolução das formulações $R, S$ e $G$, utilizando $\mathrm{HCI}$ 0,1 mol/L como meio de dissolução $(37+/-$ $\left.0,5^{\circ} \mathrm{C}\right)$, quantificação em espectrofotômetro em $250 \mathrm{~nm}$, aparatos: A) cesta, $100 \mathrm{rpm}, \mathrm{B}$ ) cesta, $50 \mathrm{rpm}, \mathrm{C})$ pá, 75 rpm e D) pá, 50 rpm.
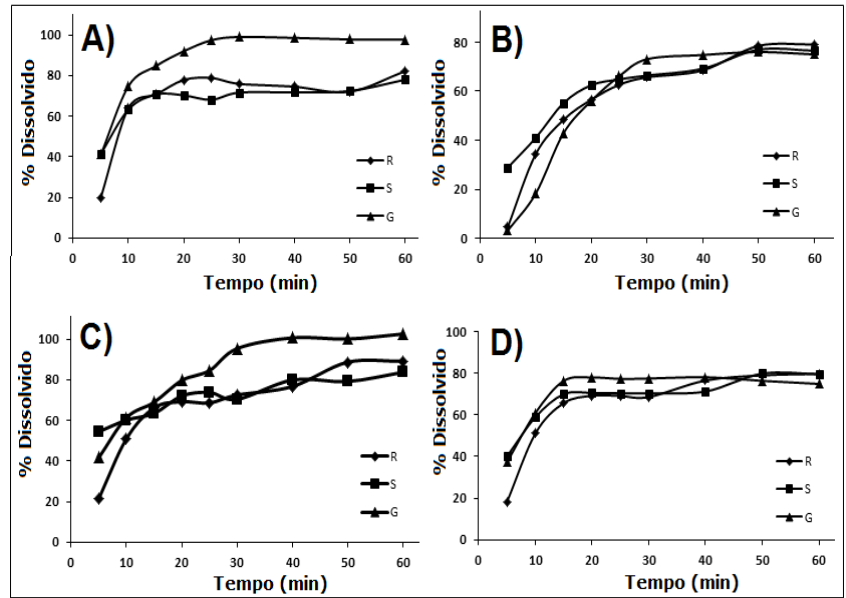

A visualização dos perfis de dissolução nos permite observar que a liberação dos comprimidos de cinarizina, $R$, $\mathrm{G}$ e $\mathrm{S}$ apresentaram um perfil de dissolução semelhante em todas as condições, porém com algumas diferenças de acordo com o aparato e velocidade de agitação utilizados.

Em menores velocidades (fig 4 B e D), os perfis foram muito semelhantes entre si, e, além disso, foram observados os menores percentuais de liberação. Em maiores velocidades de rotação (fig $4 \mathrm{~A}$ e $\mathrm{C}$ ), foi observado maiores diferenças entre as formulações, sendo que o $G$ liberou $O$ fármaco mais rapidamente. A $\mathrm{R}$ dissolveu aproximadamente $60 \%$ em 15 minutos em todas as condições. Os excipientes utilizados em $\mathrm{G}$ são os mesmos dos utilizados em $\mathrm{R}$, porém não são descritas as quantidades, e por isso, podem ocorrer essas diferenças nos perfis. A dureza, também pode influenciar no perfil de dissolução, mas nesse estudo, R, G e
S apresentaram valores de dureza muito semelhantes entre si, não sendo este um fator de influência.

De acordo com o Sistema de Classificação Biofarmacêutica (BCS), um medicamento é considerado de dissolução rápida quando não menos do que $85 \%$ do valor rotulado da substância do fármaco dissolve-se dentro de 30 minutos, usando aparato cesta a $100 \mathrm{rpm}$ e aparato pá a 50 ou $75 \mathrm{rpm}^{(13,22,23)}$.

Nesse estudo, em 30 minutos, segundo a figura 5 A) R liberou $75 \%$, S 71\%, e G 98\%; em 4 B) R liberou 65\%, S 66\% e G 73\%; em 4 C) R liberou $72 \%$, S $70 \%$ e G $95 \%$ e, em 4 D) R liberou $68 \%$, S $70 \%$ e G $77 \%$, ou seja, utilizando os aparatos pá nas velocidades de 50 (fig 4 D) e 75 (fig 4 C) rpm ou cesta na velocidade de $100 \mathrm{rpm}$ (fig 4 A), todas as formulações cumprem critérios de formulações de liberação imediata, pois liberaram praticamente $70 \%$ em $30 \mathrm{~min}$. Em cesta $50 \mathrm{rpm}$, esse critério não foi cumprido.

Durante a validação de ensaios de dissolução é importante testar diferentes aparatos em diferentes velocidades, buscando, um método que consiga discriminar as formulações, e, que atenda o critério de liberação imediata, se for este o caso $^{(13,24)}$

Os perfis de dissolução foram comparados através do fator de similaridade (f2), obtendo os valores descritos na tabela 3.

Tabela 3. Fator de similaridade ( $f 2$ ) para comparação dos perfis de dissolução das formulações R, G e S.

\begin{tabular}{lllll}
\hline Comparação & Cesta, 100 rpm & Cesṭ,, 50 rpm & Pá, 75 rpm & Pá, 50 rpm \\
\hline R x G & 36,34 & 58,06 & 39,81 & 50,79 \\
R x S & 52,91 & 52,18 & 45,59 & 54,38 \\
\hline
\end{tabular}

Os valores de f2 devem ficar entre 50 - 100 para afirmarmos que os perfis de dissolução são semelhantes ${ }^{(13)}$. Dessa forma, utilizando pá a $75 \mathrm{rpm}$, a $S$ não foi semelhante ao $\mathrm{R}$, e, utilizando cesta a $100 \mathrm{rpm}$ e pá a $75 \mathrm{rpm}$ G não foi semelhante ao R. Desse modo, pode-se considerar que o aparato pá na velocidade de $75 \mathrm{rpm}$ é o ideal para discriminar as formulações.

Equivalentes farmacêuticos são medicamentos que possuem mesma forma farmacêutica, mesma via de administração e mesma quantidade da substância ativa, podendo ou não conter excipientes idênticos. Devem cumprir com os requisitos da monografia individual da Farmacopeia Brasileira, ou com os de outros compêndios oficiais aprovados pela ANVISA $^{(13)}$. Para que um medicamento $G$ ou $S$ seja intercambiável ao $R$, é necessária a comprovação da equivalência farmacêutica e da bioequivalência. Tal fato, aliado ao cumprimento das boas práticas de fabricação e controle, fornece as bases técnicas e científicas para a intercambialidade entre o $\mathrm{G}$ ou o $\mathrm{S}$ e o respectivo medicamento $R$, visto que, nessas condições, os medicamentos apresentam a mesma eficácia clínica e o mesmo potencial para gerar efeitos $\operatorname{adversos}^{(13,25)}$.

As formulações $\mathrm{G}$ e S foram diferentes da $\mathrm{R}$ (em pá a 75 $\mathrm{rpm}$ ), pois, liberaram mais rapidamente e, neste caso, questiona-se se haveriam problemas in vivo (de eficácia terapêutica), já que se trata de uma formulação de liberação imediata. Não há na literatura, evidências que a liberação mais rápida ocasiona maiores eventos adversos bem como, muitos trabalhos que tratam a respeito do desenvolvimento de formulações a base de cinarizina, buscam uma liberação mais rápida.

Teoricamente para serem equivalentes farmacêuticos, a formulação teste deve apresentar resultados semelhantes nos ensaios de controle de qualidade, e, principalmente devem apresentar perfis de dissoluções semelhantes ao $R$, comprovados pelo valor de F2. Diante destes resultados, observa-se que $\mathrm{G}$ e $\mathrm{S}$ não são equivalentes farmacêuticos ao R.

A formulação $S$ utilizada neste estudo, não está na lista atualizada de similares intercambiáveis ${ }^{(26)}$, porém, desde a publicação da Lei no 13.235, de 29 de dezembro de 2015, que altera a Lei no 6.360, de 23 de setembro de 1976, para 
equiparar o controle de qualidade de medicamentos $\mathrm{S}$ ao de medicamentos $G$, os medicamentos $S$ devem ser equivalentes farmacêuticos ao $\mathrm{R}^{(27)}$. Além dos ensaios de qualidade, a formulação G, para ser registrada como tal, precisa realizar ensaios de bioequivalência, pois é considerada intercambiável ao R.

Em um ensaio farmacopeico de controle de qualidade de rotina, coleta-se normalmente apenas um único tempo de dissolução (e não um perfil em vários tempos). Desta forma, por não haver monografia para os comprimidos de cinarizina, todas as formulações possivelmente estariam aprovadas, pois apresentaram uma dissolução de mais de $70 \%$ em 30 minutos. Para outras monografias de comprimidos de liberação imediata, a Farmacopeia Brasileira apresenta diversos critérios de tolerância para aprovar neste ensaio de dissolução, dependendo do fármaco(12). Por exemplo, a tolerância descrita para comprimidos de liberação imediata de hidroclorotiazida é de dissolver no mínimo $60 \%$ em 30 minutos. Outro exemplo a citar-se é do ritonavir cápsulas, onde devem dissolver no mínimo $40 \%$ do fármaco em 60 minutos e no mínimo $75 \%$ do fármaco em 120 minutos.

\section{Conclusão}

O método por espectrofotometria na região do ultravioleta proposto foi desenvolvido e validado de acordo com os parâmetros de precisão, exatidão, linearidade, especificidade e robustez e por meio deste método, foi possível quantificar cinarizina em comprimidos. Não foi observada interferência dos excipientes da formulação, demonstrando adequação na análise destes comprimidos. Esta técnica é de fácil e rápida execução e pode ser aplicada no controle de qualidade rotineiro da matéria-prima e dos comprimidos de cinarizina em indústrias farmacêuticas.

As amostras apresentaram conformidade com as especificações farmacopeicas nos ensaios de doseamento, peso médio, dureza, friabilidade, desintegração e uniformidade de doses.

O ensaio de dissolução foi desenvolvido e validado em $\mathrm{HCl} 0,1 \mathrm{~mol} / \mathrm{L}$ em $\mathrm{pH} 1,2$ e mostrou linearidade, precisão, exatidão e especificidade.

Os perfis de dissolução mostraram diferenças de acordo com os aparatos e velocidades de agitação utilizados. A dissolução das formulações $R, G$ e $S$ foi maior que $70 \%$ em 30 minutos na maioria das condições testadas. Por meio do fator de similaridade, foi observado que o aparato pá a 75 rpm é a condição que discrimina melhor as formulações, pois os perfis foram diferentes. Nessa condição, tanto o G quanto o $S$, foram diferentes de $R$, ou seja, não são equivalentes farmacêuticos e consequentemente, não são intercambiáveis, devido ao fato de liberarem mais rapidamente que $\mathrm{R}$.

Os resultados obtidos mostram que os métodos desenvolvidos atendem aos requisitos de boas práticas de laboratório, pois apresentam a confiabilidade requerida para um método analítico

\section{Referências}

1. Stanley TH, Egan TD, Van Aken H. A tribute to Dr. Paul A. J. Janssen: entrepreneur extraordinaire, innovative scientist, and significant contributor to anesthesiology. Anesth Analg. 2008;106(2):451462.

2. COMMISSION BP. British Pharmacopeia. Office TS, editor. London; 2013.

3. CHEMICALIZE. www.chemizalize.org. 2015 [cited 2015 Dec 16]. Available from:

www.chemizalize.org

4. Brunton LL, Chabner B, Knollmann BC. As bases farmacológicas da terapêutica de Goodman \& Gilman. AMGH, editor. Porto Alegre; 2012.

5. Korolkovas A, França FF de AC de. DTG dicionário terapêutico Guanabara 2012/2013. Koogan G, editor. Rio de Janeiro; 2012.

6. Fabiani G, Pastro PC, Froehner C. Parkinsonism and other movement disorders in outpatients in chronic use of cinnarizine and flunarizine. Arq Neuropsiquiatr. 2004;62(3 B):784-8.

7. García-Ruiz PJ, Jiménez-Jiménez FJ, De Yébenes JG. Calcium channel blocker-induced parkinsonism: Clinical features and comparisons with Parkinson's disease. Park Relat Disord. 1998;4(4):211-4.

8. Shin HW, Chung SJ. Drug-Induced parkinsonism. J Clin Neurol. 2012;8(1):15-21.

9. Terland O, Flatmark T. Drug-induced parkinsonism: Cinnarizine and flunarizine are potent uncouplers of the vacuolar $\mathrm{H}+-$ ATPase in catecholamine storage vesicles. Neuropharmacology. 1999;38(6):879-82.

10. BRITISH PHARMACOPOEIA. Draft monograph Cinnarize Tablets. 2017 [cited 2017 Jul 10]. Available from: https://www.pharmacopoeia.com/drafttext/Cinnarizine-Tablets.pdf

11. Brasil. Resolução Da Diretoria Colegiada - RDC No 166, de 24 de julho de 2017. Ministério da Saúde - MS Agência Nacional de Vigilância Sanitária - ANVISA. 2017 [cited 2017 Aug 2]. Available from: http://portal.anvisa.gov.br/documents/10181/272156 7/RDC_166_2017_COMP.pdf/d5fb92b3-6c6b-41308670-4e3263763401

12. BRASIL. Ministério das Saúde. Agência Nacional de Vigilância Sanitária. Farmacopéia Brasileira. 5th ed. Anvisa, editor. Vol. 1, Diário Oficial da União. Brasília; 2010. 546 p. Available from:

http://www.anvisa.gov.br/hotsite/cd_farmacopeia/pdf /volume1.pdf

13. Brasil. Resolução RDC No. 31, de 11 de agosto de 2010. Agência Nacional de Vigilância Sanitária (Anvisa). 2010 [cited 2016 Jun 25]. Available from: http://portal.anvisa.gov.br/documents/33880/256807 0/res0031_11_08_2010.pdf/5e157d15-d3d5-4bb998db-5667e4d9e0c8

14. Moore JW, Flanner HH. Mathematical Comparison of Curves with an Emphasis on in Vitro Dissolution Profiles. Pharm Technol. 1996;20(6):64-74.

15. FDA. Guidance for Industry Dissolution Testing of Immediate Release Solid Oral Dosage Forms [Internet]. Vol. 4, U.S. Department of Health and Human Services Food and Drug Administration Center for Drug Evaluation and Research. 1997 [cited 2017 Jun 8]. p. 15-22. Available from:

https://www.fda.gov/downloads/drugs/guidances/ucm 070237.pdf

16. ICH - International Conference on Harmonization. Validation of Analytical Procedures: Text and Methodology Q2 (R1). ICH Steer Committee, Switz. 2005;17.

17. Gehring PAF, Santos OMM, Pereira GR, Araújo MB de, Bonfili R. Estabelecimendo de condições para ensaio de dissolução de cápsulas de cinarizina empregando planejamento fatorial. Quim Nova. 2011;34(3):45561.

18. Ratnakar NC, Prajapati BG. Formulation design and development of Cinnarizine fast disintegrating tablet. Der Pharm Sin. 2011;2(2):333-40.

19. Damodharan N, Mothilal M, Vasundhara G. Formulation and optimization of fast dissolving cinnarizine tablets. Int J PharmTech Res. 2010;2(2):1095-100.

20. Nagarwal RC, Ridhurkar DN, Pandit JK. In vitro release kinetics and bioavailability of gastroretentive cinnarizine hydrochloride tablet. AAPS PharmSciTech. 2010;11(1):294-303.

21. Kalava B, Demirel M, Yazan Y. Physicochemical characterization and dissolution properties of 
cinnarizine solid dispersions. Turkish J Pharm Sci. 2005;2(2):51-62.

22. FDA. Guidance for Industry, Waiver of in vivo bioavailability and bioequivalence studies for immediate release solid oral dosage forms based on a biopharmaceutics classification system. U.S. Department of Health and Human Services Food and Drug Administration Center for Drug

Evaluation and Research. 2015 [cited 2014 Feb 2]. p. 1-2. Available from:

https://www.fda.gov/downloads/Drugs/GuidanceCo mplianceRegulatoryInformation/Guidances/ucm070 246.pdf

23. FDA. Guidance for Industry. Dissolution Testing and Specification Criteria for Immediate-Release Solid Oral Dosage Forms Containing Biopharmaceutics Classification System Class 1 and 3 Drugs. U.S. Department of Health and Human Services Food and Drug Administration Center for Drug Evaluation and Research (CDER). 2015 [cited 2017 Aug 10]. p. 5. Available from:

https://www.fda.gov/downloads/Drugs/Guidances/ UCM456594.pdf

24. Qureshi SA. Developing Discriminatory Drug

Dissolution Tests and Profiles:Some Thoughts for Consideration on the Concept and Its Interpretation. Dissolution Technol. 2006;(November):18-23.

25. Brasil. Resolução da Diretoria Colegiada - RDC No 58, de 10 de outubro de 2014. Agência Nacional de Vigilância Sanitária (Anvisa). 2014 [cited 2016 Jun 22]. Available from:

http://portal.anvisa.gov.br/documents/33880/2568 070/rdc0058_10_10_2014.pdf/9536c1c1-29ff4405-a5f1-230335cdc37f

26. Brasil. Lista de Medicamentos Similares e seus respectivos medicamentos de referência, referência Lista de Medicamentos Similares e seus respectivos medicamentos de referência, conforme RDC 58 / 2014. 2017 [cited 2017 Sep 15]. p. http://portal.anvisa.gov.br/documents/219201/219 40. Available from:

http://portal.anvisa.gov.br/documents/33836/3527 $82 /$ Lista+de+medicamentos+similares+intercambi áveis/27d0f06c-5082-4a92-a667-08b4763a498f 